\title{
Examination of Enhancing Efficiency of Axial Gap Motor in High Speed and High Torque Region by Adopting Neodymium Bonded Magnet
}

\author{
Ren Tsunata ${ }^{* a)}$ \\ Member, Masatsugu Takemoto* Member \\ Satoshi Ogasawara** Fellow, Koji Orikawa ${ }^{* *} \quad$ Member \\ Tatsuya Saito ${ }^{* * *} \quad$ Member, Tomoyuki Ueno ${ }^{* * *} \quad$ Non-member
}

J-STAGE Advance published date : April 30, 2021

\begin{abstract}
In recent years, more than half of the electric power generated in the country is being consumed by motors. Therefore, high performance motors are desired especially for industrial applications. In addition, it is desirable to reduce motor size. Recently, motors called axial gap type have been proposed and researched to achieve both high torque and small size. Axial gap motors are generally suitable for applications requiring flat shape such as a disk. Conventional axial gap motors frequently employ Nd sintered permanent magnets (PMs) to achieve high torque. However, axial gap motors with Nd sintered PMs are not very efficient at high rotational speed due to the eddy current loss arising in the PM. Axial gap motors that use ferrite PMs have also been proposed, but torque density is low. In this paper, an axial gap motor using Nd bonded PMs is proposed to achieve high efficiency in the high-speed and high-torque region. The proposed axial gap motor using Nd bonded PM is compared with other axial gap motors employing Nd sintered PM and ferrite PM through 3D-FEA and experiments. Consequently, it was found that the Nd bonded PM is more effective in enhancing the efficiency of an axial gap motor in the high-speed and high-torque region, compared with $\mathrm{Nd}$ sintered PM and ferrite PM.
\end{abstract}

Keywords: Axial gap motor, Neodymium bonded PM, Neodymium sintered PM, Ferrite PM, Eddy current loss, Soft magnetic composite

\section{Introduction}

Currently, more than half of the electric power in Japan is consumed by motors, creating strong demand for more efficient motors. Much of that electric power is consumed by industrial motors, which often require constant torque characteristics. On the other hand, there is also increasing demand for smaller motors, but as the motors become smaller, their torque inevitably decreases. This means the motor must be made faster in order to maintain the same output.

Meanwhile, the motor load of a fan or a pump increases by the second or third power with respect to its rpm, so the higher the rotational speed, the heavier the load. In summary, higher speed is required to downsize the motor, but on the other hand, the higher the speed, the heavier the load the motor must operate under. For motors with constant torque characteristics, it is therefore important to increase efficiency in this high-speed, heavy-load region.

The application targeted in this paper is small- to medium-

a) Correspondence to: Ren Tsunata. E-mail: tsunata@okayamau.ac.jp

* Graduate School of Natural Science and Technology, Okayama University

3-1-1, Tsushimanaka, Kita-ku, Okayama 700-8530, Japan

** Graduate School of Information Science and Technology, Hokkaido University

Kita 14, Nishi 9, Kita-ku, Sapporo, Hokkaido 060-0814, Japan

*** Sumitomo ElectricIndustries, Ltd.

Itami, Hyogo 664-0016, Japan capacity water pumps, which account for a large percentage of industrial motors. Since cavitation is more likely to occur above $6000 \mathrm{rpm}$ in small- to medium-capacity water pumps, in this paper, we define the high-speed region as $6000 \mathrm{rpm}$.

In addition, to increase the added value of the system that incorporates the motor, it is often desirable for the motor to have a flat profile. For this reason, the axial gap motor has been attracting attention ${ }^{(1)-(4)}$. The flat design of the axial gap motor makes it possible to increase the torque by increasing the surface area of the magnet.

Many of conventional axial gap motors have used neodymium (Nd) sintered permanent magnets (PMs) to achieve high torque, since this magnet material exhibits the highest residual magnetic flux density, as shown in Fig. 1. However, axial gap motors employing Nd sintered PMs are not very efficient in the high-speed region due to the eddy current loss arising in the $\mathrm{PM}^{(5)-8)}$, resulting in a motor with high torque density but poor efficiency.

To overcome this problem, various measures have been proposed to suppress the eddy current loss arising in the PM of conventional axial gap motors, such as adopting an interior magnet rotor structure ${ }^{(9)}$ or optimizing the magnet divi$\operatorname{sion}^{\left({ }^{(1)}\right)}$. However, these countermeasures are costly and make the rotor structure more complicated. Even if they can be applied to large capacity motors, they are difficult to apply to small- to medium-capacity industrial motors, which are under strict cost constraints.

From the above, conventional axial gap motors using $\mathrm{Nd}$ 


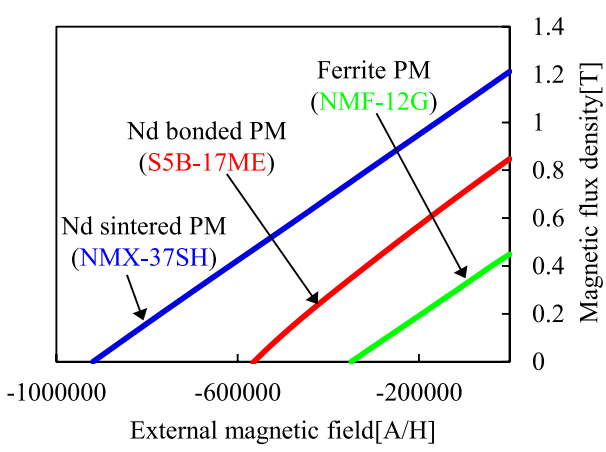

Fig. 1. $B-H$ curves of $\mathrm{Nd}$ sintered, bonded and ferrite PMs

sintered PMs are mostly used in the low-speed, heavy-load region. As a countermeasure to overcome the eddy current loss in Nd sintered PM, Many research has also been conducted on axial gap motors using ferrite PMs, which have a much smaller residual magnetic flux density (about 1/3) than Nd sintered PMs, as shown in Fig. $1^{(11)(12)}$. Axial gap motors employing ferrite PMs exhibit high efficiency in the lightload region over a wide range of speeds, but require a high current density to handle heavy loads, and suffer reduced efficiency at heavy loads due to high copper loss.

As noted above, the conventional axial gap motor has the problem of reduced efficiency in the high-speed, heavy-load region. The aim of this paper is to improve the efficiency of axial gap motors in the high-speed, heavy-load region by adopting an Nd bonded PM. To demonstrate the effectiveness of our proposed method, we performed 3D-FEA analysis and experiments on prototype motors to compare our proposed axial gap motor using a $\mathrm{Nd}$ bonded $\mathrm{PM}$ against comparable setups using a ferrite PM and an Nd sintered PM. The results confirmed that the proposed motor exhibits the highest efficiency in the target high-speed, heavy-load region.

\section{Structure and Materials of the Proposed Mo- tor}

\subsection{Structure and Parameters of the Proposed Motor}

Table 1 shows the basic specifications and Fig. 2 shows the structure of the proposed axial gap motor, which employs an Nd bonded PM. As shown in Fig. 2, the motor has a shaft length $\mathrm{L}$ of $41.3 \mathrm{~mm}$, an outer diameter $\mathrm{D}$ of $110 \mathrm{~mm}$, and an aspect ratio (L/D), calculated by dividing $\mathrm{L}$ by $\mathrm{D}$, of 0.375 , giving the motor a flat shape. To reduce cogging torque, a fractional-slot construction with 10 poles and 12 slots was adopted $^{(13)-(15)}$. A relatively large air gap of $1.0 \mathrm{~mm}$ on each side was adopted to eliminate the need to fine-tune the axial position of the rotor by a shim or the like when the actual motor is manufactured.

Figure 3(a) shows a detailed model of the rotor. To reduce costs, we adopted a surface permanent magnet (SPM) configuration with a coreless rotor structure in which only the permanent magnets are embedded into the non-magnetic PM holder. The PM holder is made of non-magnetic SUS304 and is provided with slits to reduce eddy current loss. ${ }^{(16)}$ The $\mathrm{Nd}$ bonded PM (S5B-17ME, Aichi Steel Co., Ltd.) indicated in Fig. 1 was used as the PM of the proposed motor. The SPM structure is known to be susceptible to eddy current loss ${ }^{(17)-(19)}$, but we suspected that this problem could be overcome by the
Table 1. Main parameters of the proposed axial gap motor

\begin{tabular}{c|c}
\hline Parameter & Value \\
\hline \hline Total magnet weight & $89.2 \mathrm{~g}$ \\
\hline Occupied volume of motor & $0.392 \mathrm{~L}$ \\
\hline The number of poles & 10 poles \\
\hline The number of slots & 12 slots \\
\hline Coil space factor & $40 \%$ \\
\hline DC-bus voltage & $282 \mathrm{~V}$ \\
\hline
\end{tabular}

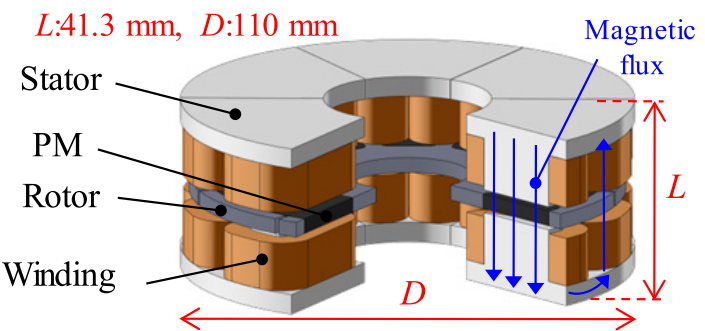

Fig. 2. Configuration of the proposed axial gap motor using $\mathrm{Nd}$ bonded PM

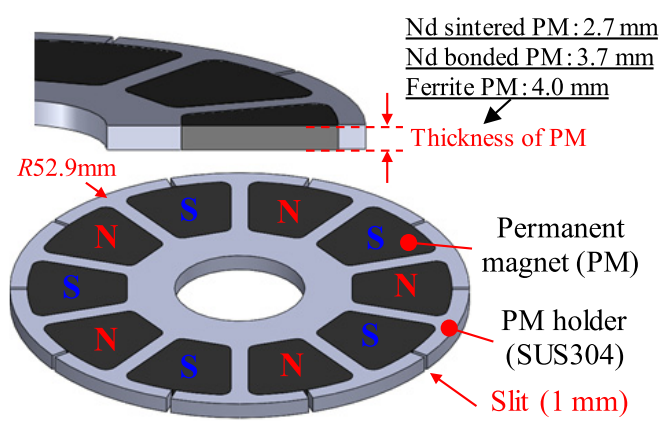

(a) Rotor structure

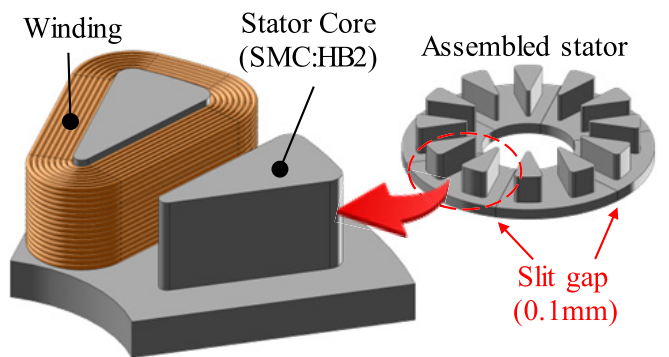

(b) Stator structure

Fig. 3. Each component of the proposed axial gap motor

Nd bonded PM, which is less likely to generate eddy current loss compared to the Nd sintered PM.

Figure 3(b) shows a detailed model of the stator. For the stator core, we adopted a compacted iron core based on soft magnetic composite materials (SMCs) ${ }^{(20)(21)}$, yielding an easy-to-mold stator core capable of handling 3D magnetic flux flow. The SMC used was HB2 (Sumitomo Electric Industries, Ltd.), which has low iron loss characteristics. As shown in the figure, the stator core is divided into six segments. To account for the effects of this segmented core, we used 3D-FEA analysis to run simulations with a slit gap of $0.1 \mathrm{~mm}$ between each segment.

2.2 Characteristics of Each PM Figure 1 shows the $B$ - $H$ curves for the Nd bonded PM used in the proposed motor as well as a Nd sintered PM (NMX-37SH, Hitachi Metal, Ltd.) and a ferrite PM (NMF-12G, Hitachi Metal, Ltd.). The 
residual magnetic flux density of the Nd bonded PM is 0.71 times the Nd sintered PM and 1.8 times the ferrite PM, yielding a curve intermediate between the other two PMs. Table 2 shows the electrical conductivity and mass density of the three PMs. The electrical conductivity of the Nd bonded PM and the ferrite PM is about $1 / 100,000$ of that of the $\mathrm{Nd}$ sintered PM $(6.25 \times 105 \mathrm{~S} / \mathrm{m})$, which explains why eddy current losses are less likely to occur. The Nd sintered PM had the greatest mass density, 1.49 times greater than the ferrite PM, which had the smallest density. On the other hand, there is little difference in mass density between the Nd bonded PM and the ferrite PM.

\section{Analysis of the Proposed Motor Using the Nd Bonded PM}

In addition to the proposed motor using the Nd bonded PM, for comparison, we also analyzed models where we swapped in the Nd sintered PM and ferrite PM shown in Fig. 1. In this study, the total weight of the three magnet materials was kept constant at $89.2 \mathrm{~g}$. The reason for this is that the cost of magnets is generally determined by weight, and a comparison by weight is easier to understand from a cost perspective for motors that are to be mass produced. We therefore varied the magnet volume according to the densities shown in Table 2. Accordingly, as shown in Fig. 3(a), the thickness of the $\mathrm{Nd}$ sintered PM (the densest) was $2.7 \mathrm{~mm}$, the thickness of the Nd bonded PM used in the proposed motor was $3.7 \mathrm{~mm}$, and the thickness of the ferrite PM was $4.0 \mathrm{~mm}$. The stator and gap lengths were the same for all three models.

$3 \mathrm{D}$ analysis is carried out at the following three operating points:

- High-speed heavy-load: $6000 \mathrm{rpm}, 1.15 \mathrm{Nm}$

- Low-speed heavy-load: $1000 \mathrm{rpm}, 1.15 \mathrm{Nm}$

- High-speed light-load: $6000 \mathrm{rpm}, 0.23 \mathrm{Nm}$

We defined the high-speed region as $6000 \mathrm{rpm}$ because cavitation is more likely to occur above $6000 \mathrm{rpm}$ in small- to medium-capacity water pumps, which account for a large percentage of the industrial motor applications targeted in this paper ${ }^{(22)(23)}$. In terms of torque, a heavy-load is defined as $1.15 \mathrm{Nm}$. This value is obtained at a current density of $4 \mathrm{Arms} / \mathrm{mm}^{2}$ (2.51 Arms) on the Nd bonded PM model proposed in this paper. This current density is a value that allows the motor to operate continuously without cooling. We carried out the comparison by inputting different currents to each motor to match the torques. The analysis was performed by 3D-FEA using the JMAG-Designer software developed by JSOL Corporation.

3.1 Comparison of Torque Waveforms and Magnetic Flux Density Distributions Figure 4 shows the instantaneous torque waveform results for the proposed motor using the $\mathrm{Nd}$ bonded PM as well as the models using the $\mathrm{Nd}$ sintered PM and ferrite PM. The operating point of each motor is $6000 \mathrm{rpm}$ with a torque of $1.15 \mathrm{Nm}$ and a magnet temperature of $80^{\circ} \mathrm{C}$.

As shown in Fig. 4, the average torque of each motor is $1.15 \mathrm{Nm}$. However, the torque ripple differs depending on the residual magnetic flux density of the PM. The model with the Nd sintered PM indicates the greatest ripple at $12.4 \%$. The ferrite PM model, which has the lowest residual magnetic flux density, has the smallest torque ripple at $2.52 \%$,
Table 2. Some characteristics of three PMs

\begin{tabular}{c|c|c|c}
\hline & $\begin{array}{c}\text { Nd bonded } \\
\text { magnet }\end{array}$ & $\begin{array}{c}\text { Nd sintered } \\
\text { magnet }\end{array}$ & $\begin{array}{c}\text { Ferrite } \\
\text { magnet }\end{array}$ \\
\hline \hline $\begin{array}{c}\text { Electric } \\
\text { conductivity }\end{array}$ & $14.3 \mathrm{~S} / \mathrm{m}$ & $\begin{array}{c}6.25 \times \\
10^{5} \mathrm{~S} / \mathrm{m}\end{array}$ & $0.10 \mathrm{~S} / \mathrm{m}$ \\
\hline Mass density & $5500 \mathrm{~kg} / \mathrm{m}^{3}$ & $7600 \mathrm{~kg} / \mathrm{m}^{3}$ & $5100 \mathrm{~kg} / \mathrm{m}^{3}$ \\
\hline
\end{tabular}

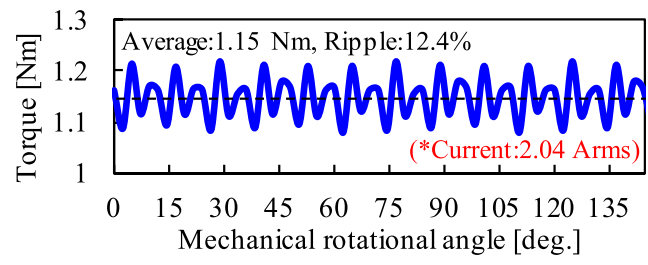

(a) Nd sintered PM model at 3.25 Arms $/ \mathrm{mm}^{2}$ (2.04 Arms)

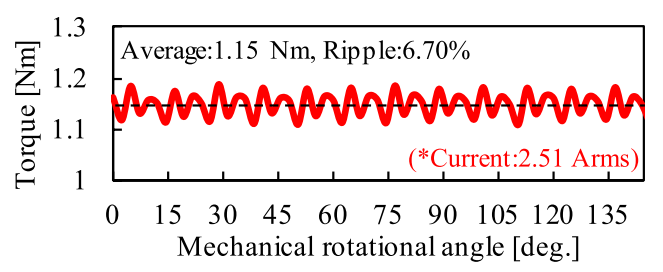

(b) Nd bonded PM model at $4.0 \mathrm{Arms} / \mathrm{mm}^{2}$ (2.51 Arms)

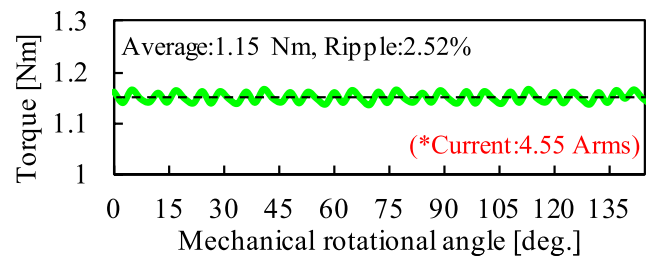

(c) Ferrite PM model at $7.25 \mathrm{Arms} / \mathrm{mm}^{2}$ (4.55 Arms)

Fig. 4. Instantaneous torque waveforms of three axial gap motors having different permanent magnet materials (@6000 rpm, 1.15 Nm)

and the proposed Nd bonded PM model has an intermediate torque ripple value of $6.70 \%$.

The residual magnetic flux density of the $\mathrm{Nd}$ sintered PM is 1.44 times that of the $\mathrm{Nd}$ bonded PM. The current required to output $1.15 \mathrm{Nm}$ of torque was $2.04 \mathrm{Arms}$ for the $\mathrm{Nd}$ sintered PM and 2.51 Arms for the Nd bonded PM. The current for the Nd bonded PM model is 1.23 times higher than the sintered model, which is smaller than the corresponding difference in residual magnetic flux density. The following two reasons likely explain why the difference in the currents is smaller than the difference in the residual magnetic flux densities: (1) The thickness of the Nd bonded PM is greater than the Nd sintered PM due to their difference in mass density; and (2) Significant eddy current loss occurs in the Nd sintered PM and energy that would normally contribute to the torque is lost.

Figure 5 shows the magnetic flux density distributions in the stator core of each motor. Figure 6 is a graph of the magnetic flux density distribution on the monitoring line from point A to point B on the stator core. Both figures show the results of the analysis at an electrical angle of 0 deg. In Fig. 6 in particular, in order to evaluate the difference in magnetic flux density due to the differences in the magnet material, the observation point is the starting point of the U-phase teeth where the magnetic flux is maximized when the electric angle is $0 \mathrm{deg}$. Since the axial gap motor under investigation has 


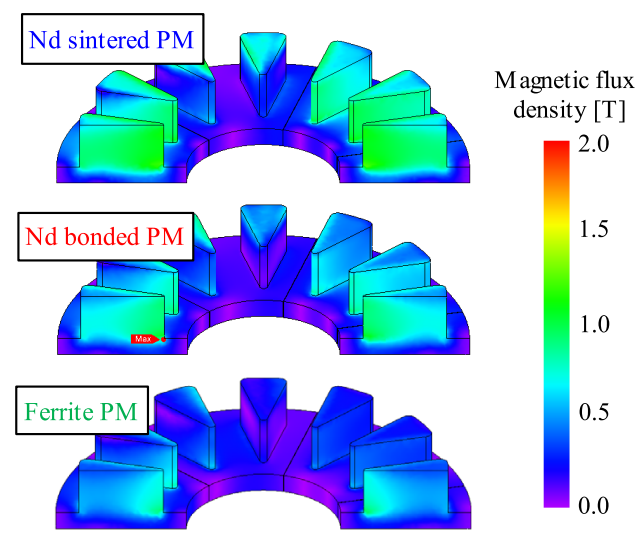

Fig. 5. Magnetic flux density distributions in the stator core of each motor $(6000 \mathrm{rpm}, 1.15 \mathrm{Nm}$, electrical angle $=0$ deg.)

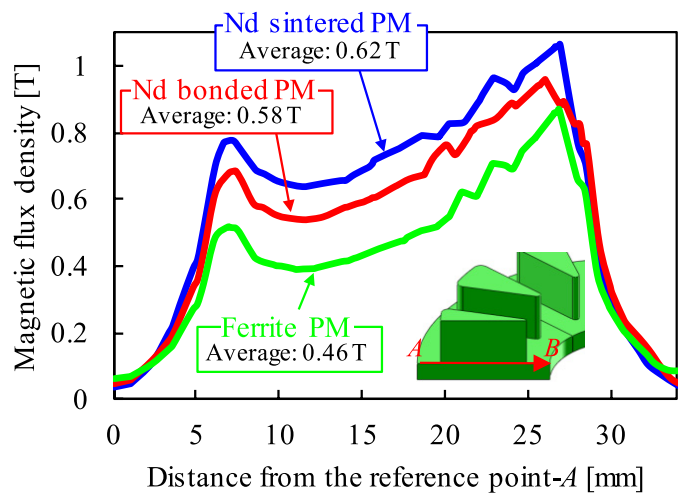

Fig. 6. Magnetic flux density on the monitoring line from $A$ to $B(6000 \mathrm{rpm}, 1.15 \mathrm{Nm}$, electrical angle $=$ 0 deg.)

a wide air gap length and a coreless rotor structure, the magnetic resistance of the proposed motor is large. The dominant factor is therefore the magnetic flux generated by the PM. This is why there is a difference in the magnetic flux density among the models even though their output is the same. The figure shows that the higher the residual magnetic flux density of the PM, the higher the magnetic flux density of the stator.

3.2 Comparison of Loss and Efficiency Figure 7 shows the efficiency and a breakdown of the losses of each axial gap motor. The analysis conditions are the same as in the previous section: the high-speed heavy-load region at $6000 \mathrm{rpm}$ and $1.15 \mathrm{Nm}$. The copper loss is calculated using the resistance value measured in the prototype motors described below. The motor with the highest efficiency was the proposed motor, which achieved an efficiency of $94.8 \%$. The next most efficient was the ferrite PM model, with an efficiency of $91.0 \%$. It also required the largest current to output $1.15 \mathrm{Nm}$. The low residual magnetic flux density of the ferrite PM model means the current needs to be increased under heavy loads, resulting in very large copper losses.

The total loss of the model with the Nd sintered PM is $114 \mathrm{~W}$, which was 2.9 times the loss of the proposed motor. As a result, the efficiency of the $\mathrm{Nd}$ sintered $\mathrm{PM}$ is $86.7 \%$, which is 8.1 percentage points lower than the $94.8 \%$ efficiency of the proposed motor. Of the total loss of $114 \mathrm{~W}$, iron loss generated in the rotor, in other words eddy current

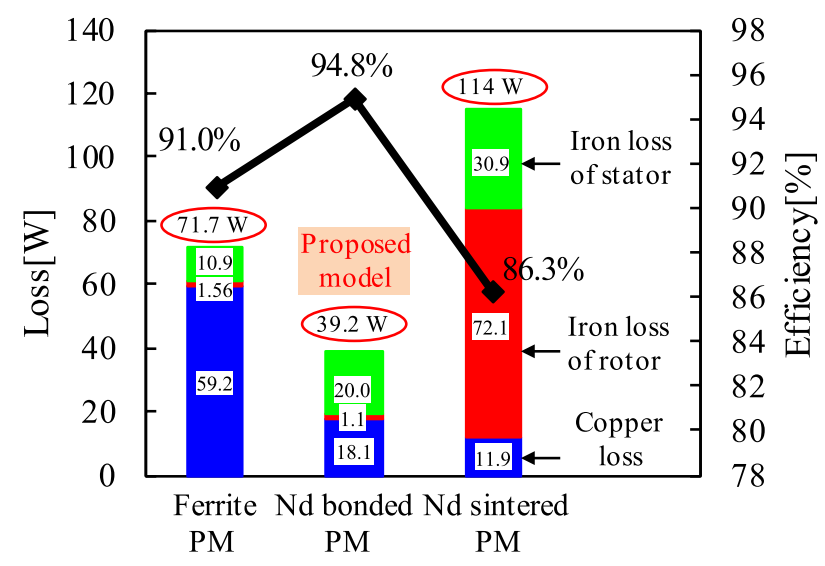

Fig. 7. The efficiency and breakdown of losses of three axial gap motors at high speed and high torque point $(6000 \mathrm{rpm}, 1.15 \mathrm{Nm})$

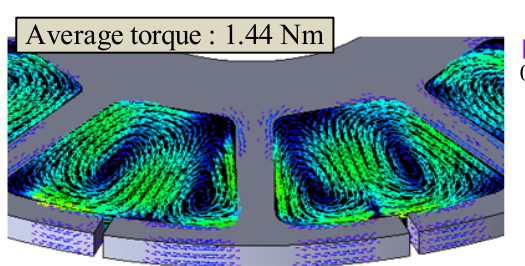

Eddy current density $\left[\mathrm{A} / \mathrm{mm}^{2}\right]$

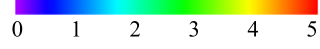

Eddy current loss Total: $73.1 \mathbf{W}$ $\rightarrow$ Magnet: $71.8 \mathrm{~W}$ $\hookrightarrow$ PM holder:1.30 W

(a) $\mathrm{Nd}$ sintered PM model at $4.0 \mathrm{Arms} / \mathrm{mm}^{2}$ (2.51 Arms)
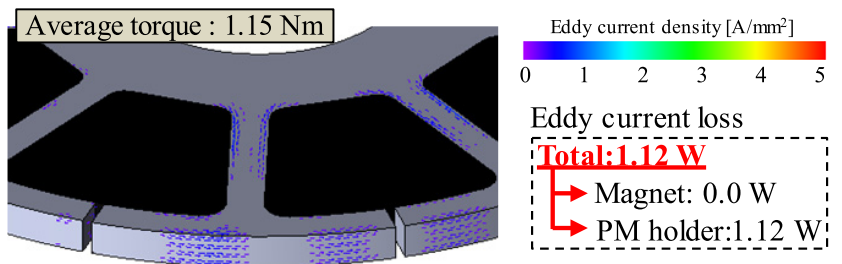

(b) The proposed Nd bonded PM model at $4.0 \mathrm{Arms} / \mathrm{mm}^{2}$ (2.51 Arms)

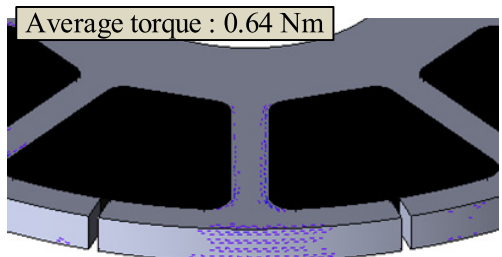

Eddy current density $\left[\mathrm{A} / \mathrm{mm}^{2}\right]$

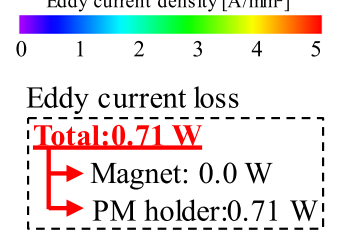

(c) Ferrite PM model at $4.0 \mathrm{Arms} / \mathrm{mm}^{2}$ (2.51 Arms)

Fig. 8. The eddy current density distributions in rotor of axial gap motors under same current $\left(4.0 \mathrm{Arms} / \mathrm{mm}^{2}\right)$ at $6000 \mathrm{rpm}$

loss, accounts for $72.1 \mathrm{~W}(63.2 \%)$.

Figure 8 shows the eddy current density distribution generated in the rotor of each axial gap motor model. The analysis conditions in Fig. 8 are $6000 \mathrm{rpm}$ and $4 \mathrm{Arms} / \mathrm{mm}^{2}$. In other words, comparison is carried out under same magnet motive force because the stator is the same. In the case of the ferrite $\mathrm{PM}$ model and the proposed motor using the $\mathrm{Nd}$ bonded $\mathrm{PM}$, the electrical conductivity of the magnet is extremely small, so the eddy current loss in the magnet is $0.0 \mathrm{~W}$, and the only eddy current loss occurs in the PM holder. This loss was $0.71 \mathrm{~W}$ for the model using ferrite PM and $1.12 \mathrm{~W}$ for the proposed model using $\mathrm{Nd}$ bonded PM, respectively. In contrast, in the model with the Nd sintered PM, the eddy current loss in the magnet was $71.8 \mathrm{~W}$, which accounts for the majority of the loss. Consequently, in the high-speed region 


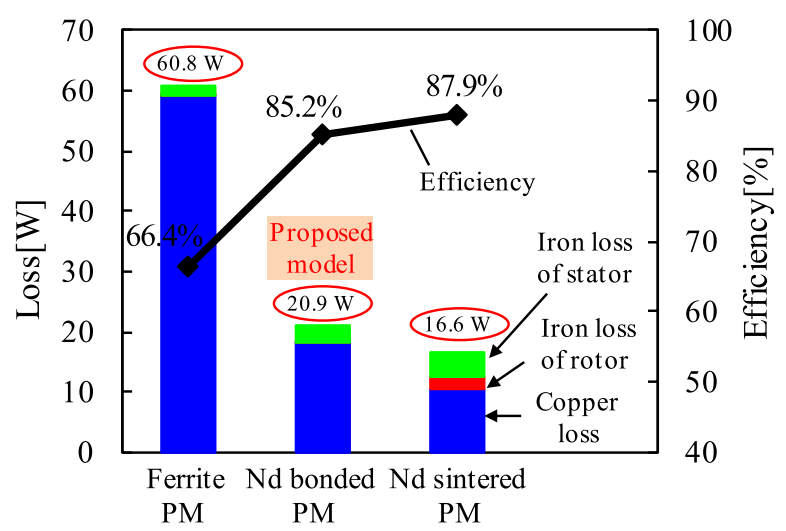

(a) Low rotational speed and high torque operating point (@1000 rpm, 1.15 Nm)

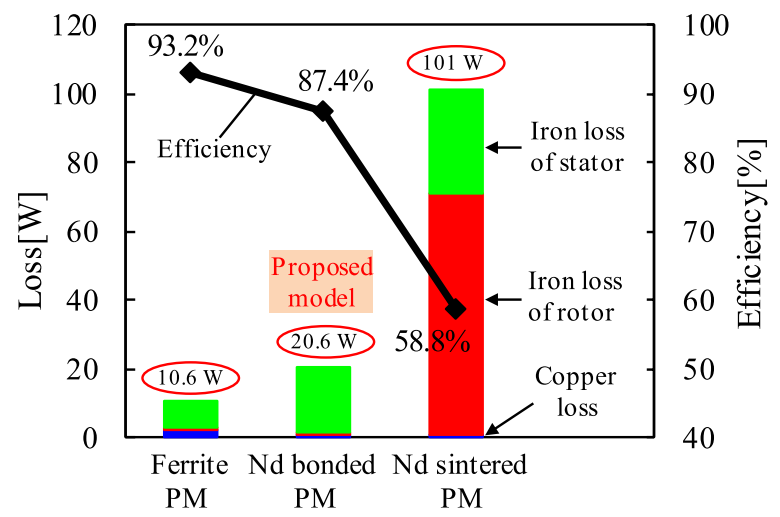

(b) High rotational speed and low torque operating point (@6000 rpm, $0.23 \mathrm{Nm}$ )

Fig. 9. Each loss and the efficiency of three axial gap motors in different points

at $6000 \mathrm{rpm}$, the axial gap motor using the $\mathrm{Nd}$ sintered PM has a large eddy current loss in the magnet, resulting in an inefficient motor with high heat generation in the rotor.

On the other hand, in the proposed motor and the ferrite PM model, there is almost no eddy current loss in the magnet, which keeps the rotor iron loss low and allows high efficiency to be achieved in the high-speed region. However, the ferrite PM model has a lower residual magnetic flux density than the Nd bonded PM, resulting in higher current and increased copper loss under heavy loads. The proposed motor using the $\mathrm{Nd}$ bonded PM is therefore the most efficient in the high-speed heavy-load region.

Figure 9 shows the respective losses and efficiency of each model in (a) the low-speed heavy-load region (1000 rpm, $1.15 \mathrm{Nm}$ ) and (b) the high-speed light-load region (6000 rpm, $0.23 \mathrm{Nm}$ ). In the high-speed heavy-load region shown in Fig. 7, the proposed motor indicates the highest efficiency, whereas in the low-speed heavy-load region shown in Fig. 9(a), the model with the Nd sintered PM achieved the highest efficiency at $87.9 \%$. In the high-speed region, the PM's eddy current loss is significant. In the low-speed region, the PM's eddy current loss is suppressed, and high residual magnetic flux density of the Nd sintered PM can contribute to increasing the efficiency. In contrast, the ferrite PM model has very large copper loss compared to the other models in the low-speed heavy-load region, reducing its efficiency to $66.4 \%$. The proposed motor achieves an efficiency of $85.2 \%$ in the low-speed heavy-load region because there is little eddy current loss generated in the rotor and its copper loss is much lower than the ferrite PM model.

In the high-speed light-load region shown in Fig. 9(b), the ferrite PM model achieved the highest efficiency at $93.2 \%$. This is because the ferrite PM has almost no eddy current loss and low residual magnetic flux density, so the iron loss generated in the stator is lower than in the other models. On the other hand, the model with the Nd sintered PM achieved an efficiency of $58.8 \%$ because the eddy current loss generated in the magnet is significantly higher compared to other models due to its higher rotational speed. This means that $28.5 \%$ of the input power is lost to eddy current loss. The proposed motor has more iron loss at the stator than the ferrite model due to the higher residual magnetic flux density of the magnet, but the magnet generates no eddy current loss, resulting in a high efficiency of $87.4 \%$.

Figure 7 and Fig. 9 show that different models achieved the highest efficiency in each operating region. However, in the high-speed heavy-load region (6000 rpm, $1.15 \mathrm{Nm}$ ), which is the focus of this paper, the highest efficiency is achieved by the proposed motor using the Nd bonded PM.

\section{Comparison of the Characteristics of Three Prototype Motors Based on Experimental Re- sults}

4.1 Appearance and Characteristics of the Prototype Motors In this section, we compare the characteristics of axial gap motors using the three different PM materials based on the results of experiments on prototype motors. Each prototype motor was load tested using the equipment shown in Fig. 10. Figure 11 shows a photograph of the stator of the prototype motor. SMC is used for the core, and the winding uses a concentrated winding construction. Figure 12 shows the three prototype rotors employing the three different magnet materials. As noted above, each rotor has a uniform magnet weight of $89.2 \mathrm{~g}$, but the rotor thicknesses are different depending on the mass density of the magnet being used. The thicknesses of the Nd sintered PM, Nd bonded PM, and ferrite PM rotors are 2.7, 3.7, and $4.0 \mathrm{~mm}$, respectively, with the thickest being the ferrite PM rotor. The same stator is used for all the axial gap motors, and the air gap length is kept constant at $1 \mathrm{~mm}$ on each side in three motors. Therefore, the shaft length of each prototype differs depending on the thickness of the rotor.

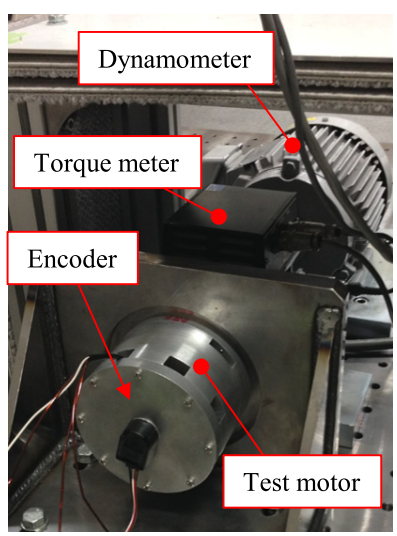

Fig. 10. Configuration of the experimental system 


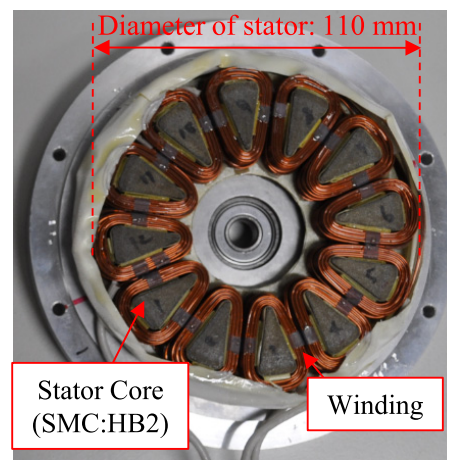

Fig. 11. The prototyped Stator employing SMC cores of axial gap motor

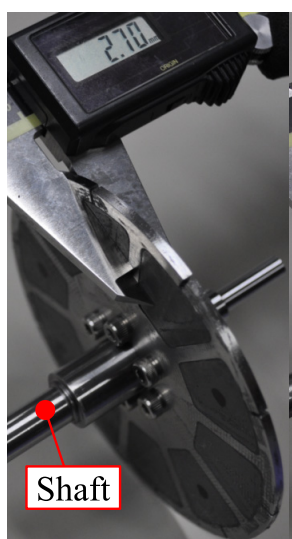

(a) $\mathrm{Nd}$ sintered $\mathrm{PM}$

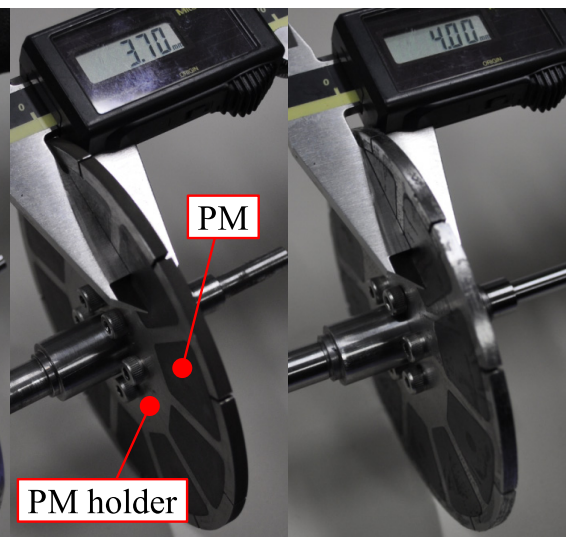

(b) Nd bonded PM (c) Ferrite PM

Fig. 12. Three prototyped rotors employing different materials

\subsection{Experimental Results}

Figure 13 shows a graph of the torque increase versus current density for the prototypes using each rotor at $1000 \mathrm{rpm}$. In each model, the current is increased until the torque reached $1.25 \mathrm{Nm}$. This produced a torque coefficient for each motor, indicating the relative increase in torque at each current density. The resulting torque coefficients for the Nd sintered PM, Nd bonded PM, and ferrite $\mathrm{PM}$ models are $0.42,0.33$, and $0.18 \mathrm{Nm} \cdot \mathrm{mm}^{2} /$ Arms, respectively. The higher the residual magnetic flux density, the higher the torque coefficient. However, the difference in residual magnetic flux density is not directly reflected in the torque performance, as indicated by the fact that the residual magnetic flux density of the $\mathrm{Nd}$ sintered $\mathrm{PM}$ is 1.43 times greater that of the Nd bonded PM, but the torque coefficient is only 1.27 times greater. This may be due to the fact that the rotor with the $\mathrm{Nd}$ sintered PM is $1 \mathrm{~mm}$ thinner than the rotor with the Nd bonded PM, as shown in Fig. 12.

Figure 14 shows the copper loss map of each axial gap motor. The maximum current densities required to match the power output ranges are 3.2, 4.0, and $7.0 \mathrm{Nm} \cdot \mathrm{mm}^{2} /$ Arms for the Nd sintered PM, Nd bonded PM, and ferrite PM models, respectively. Figure 14 shows that, for all three models, the copper loss is greatest when the maximum current is applied. Since the three models have different maximum current densities due to the difference in torque coefficients, the maximum copper losses are also different: $11.8 \mathrm{~W}, 18.1 \mathrm{~W}$, and $53.4 \mathrm{~W}$, respectively. The model using the ferrite PM has the greatest copper loss, about 4.5 times larger than the model with the Nd sintered PM. This is because the ferrite model

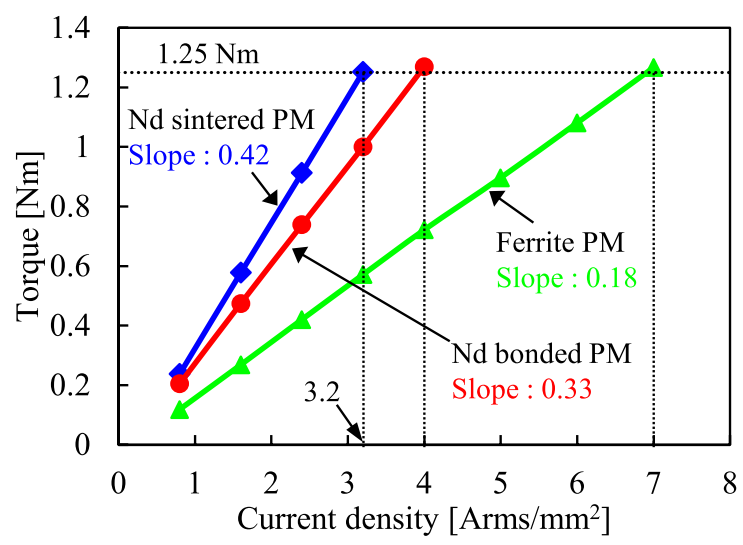

Fig. 13. The average torque vs. the current density of three prototyped axial gap motors at $1000 \mathrm{rpm}$

has a torque coefficient that is about 0.43 times the model with the Nd sintered PM, so it requires a larger current to output an equivalent torque. Examining the three maps in Fig. 14, only the model with the Nd sintered PM exhibits a decrease in torque at the same current density as the number of revolutions increases. In the model with the $\mathrm{Nd}$ sintered $\mathrm{PM}$, the torque decreases by about $17.3 \%$ as the motor speed increases from $1000 \mathrm{rpm}$ to $6000 \mathrm{rpm}$. This is because the energy that would otherwise contribute to the torque is lost as eddy current loss in the magnet.

The proposed motor with the $\mathrm{Nd}$ bonded PM has a torque coefficient that is 0.79 times the model with the $\mathrm{Nd}$ sintered $\mathrm{PM}$, which means its maximum current density is higher, and its maximum copper loss is about 1.5 times larger. However, as with the ferrite PM model, there is no eddy current loss in the magnet, so the generated torque does not decrease even when the rpm rises, allowing a constant torque operating range to be maintained.

Figure 15 shows the iron loss maps obtained experimentally for each model. All models exhibited maximum iron loss at $6000 \mathrm{rpm}$, the highest speed. The model using the $\mathrm{Nd}$ sintered PM has the largest maximum iron loss among the three models at $139.3 \mathrm{~W}$. This is because only the Nd sintered PM generates eddy current losses, and because the magnetic flux density of its stator is the highest among the models, as shown in Fig. 5 of the analysis results. On the other hand, the maximum iron loss of the ferrite PM model is the smallest at $23.1 \mathrm{~W}$, which is about 0.17 times the value of the model with the Nd sintered PM. This is because its magnet has the smallest residual magnetic flux density, which means its stator has the smallest magnetic flux density. The proposed $\mathrm{Nd}$ bonded PM motor also exhibits extremely smaller eddy current loss in the magnet. Its maximum iron loss is $32.0 \mathrm{~W}$, about 0.23 times the value for the $\mathrm{Nd}$ sintered PM model.

Figure 16 shows the total loss map for each model, including the copper loss in Fig. 14 and the iron loss in Fig. 15. Note that mechanical loss is excluded. The mechanical loss is measured by fabricating a dummy rotor of only stainless steel (SUS304) with the same total weight and moment of inertia around the rotating shaft as the actual rotor. As the total loss map shows, the maximum total losses are $146.5 \mathrm{~W}, 45.7 \mathrm{~W}$, and $75.9 \mathrm{~W}$ for the Nd sintered PM, Nd bonded PM, and ferrite PM models, respectively. All models show maximum 


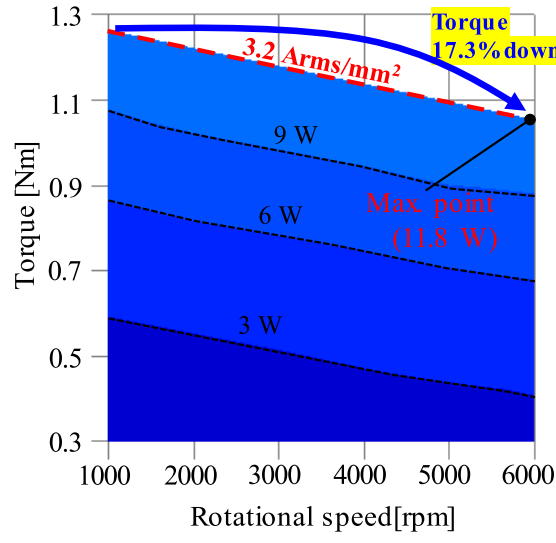

(a) Nd sintered PM model

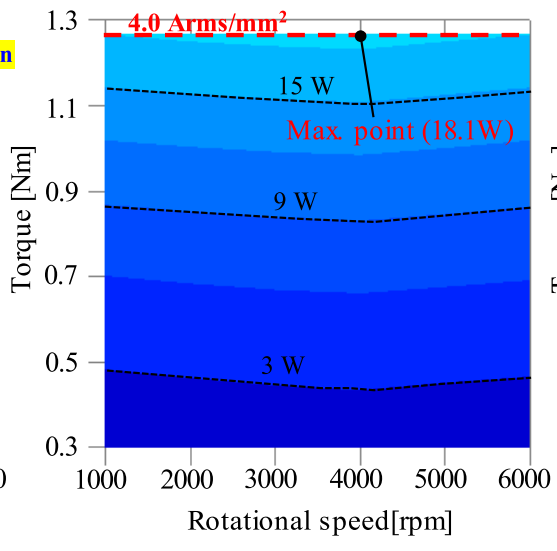

(b) Nd bonded PM model (the proposed motor)

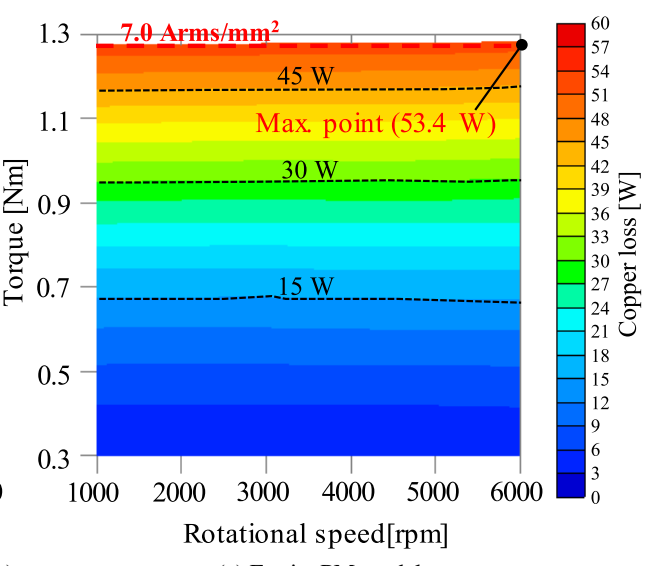

(c) Ferrite PM model

Fig. 14. Copper loss maps of three prototyped axial gap motors using different magnet materials

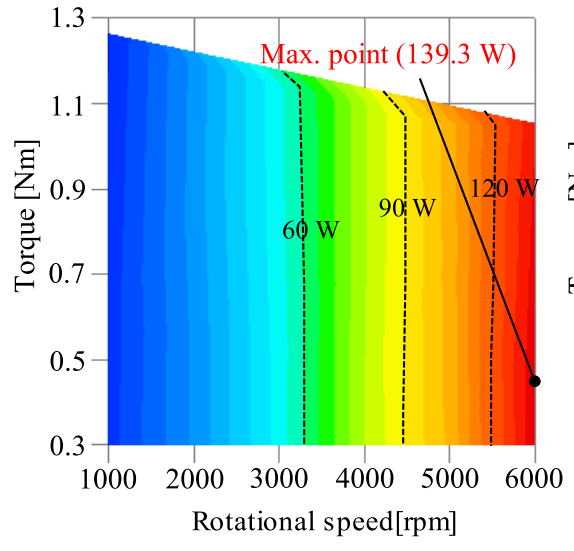

(a) Nd sintered PM model

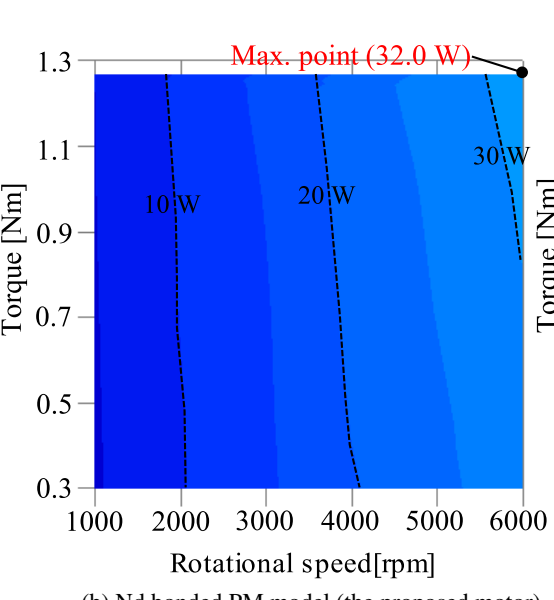

(b) Nd bonded PM model (the proposed motor)

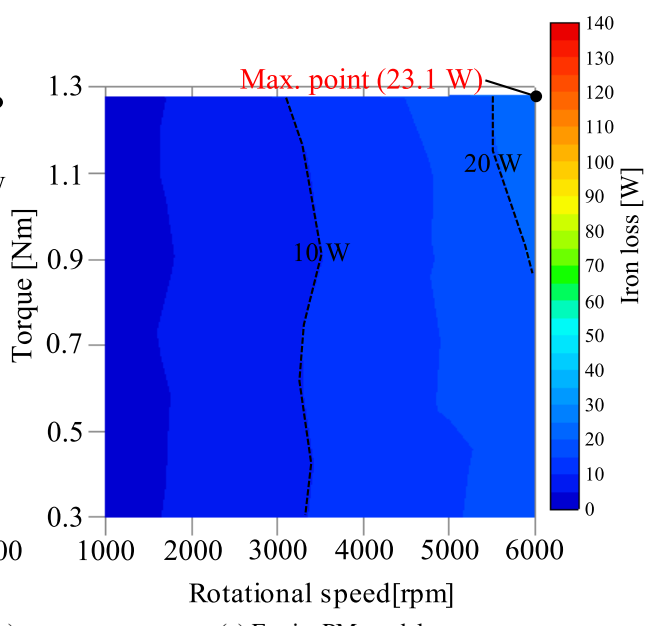

(c) Ferrite PM model

Fig. 15. Iron loss maps of three prototyped axial gap motors using different magnet materials

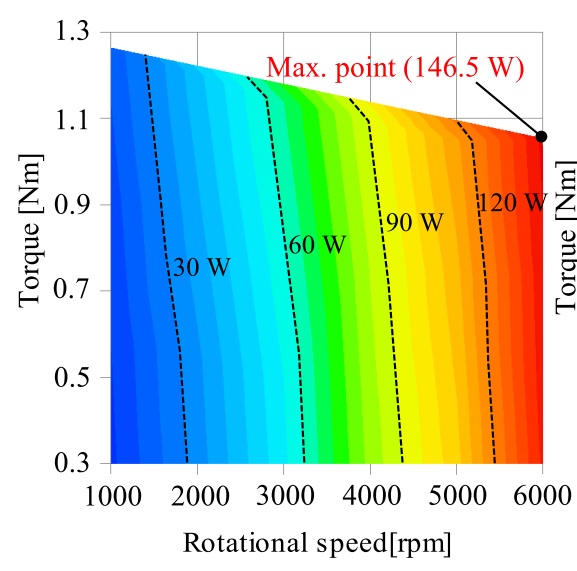

(a) Nd sintered PM model

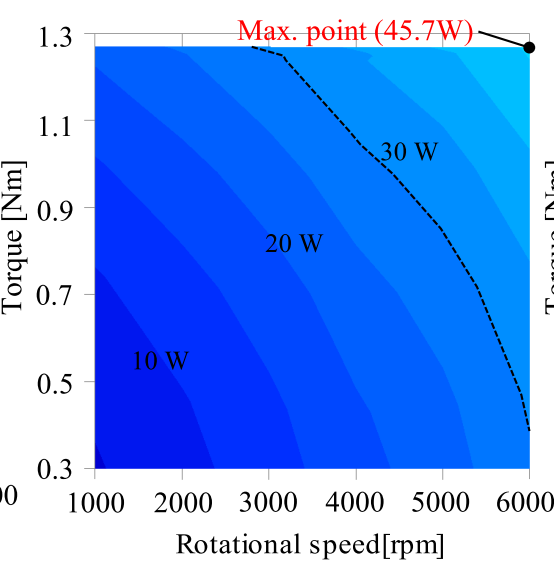

(b) Nd bonded PM model (the proposed motor)

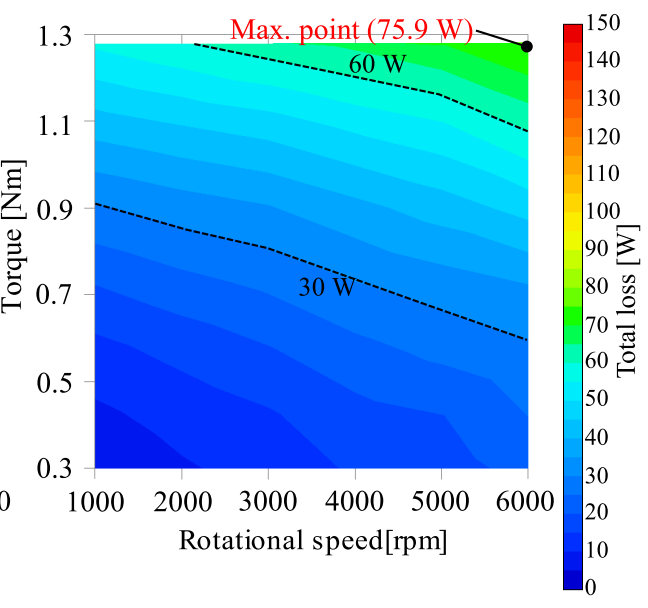

(c) Ferrite PM model

Fig. 16. Total loss maps of three prototyped axial gap motors using different magnet materials

loss in the high-speed heavy-load region $(6000 \mathrm{rpm}, 3.2$ to 7.0 Arms $/ \mathrm{mm}^{2}$ ).

The proposed motor with the Nd bonded PM has the smallest total loss at $45.7 \mathrm{~W}$, which is about 0.31 times the total loss of the model with the Nd sintered PM. This is because the proposed motor has a torque coefficient 1.83 times that of the ferrite PM model, which suppresses copper loss in the heavy-load region. In addition, the eddy currents in the magnet are almost non-existent, which suppresses the increase in iron loss in the high-speed region.

The model with the Nd sintered PM has the smallest copper loss due to its low current, but its total loss was $146.5 \mathrm{~W}$, the highest of all the models, due to the high iron loss in the high-speed region. On the other hand, the ferrite PM model 


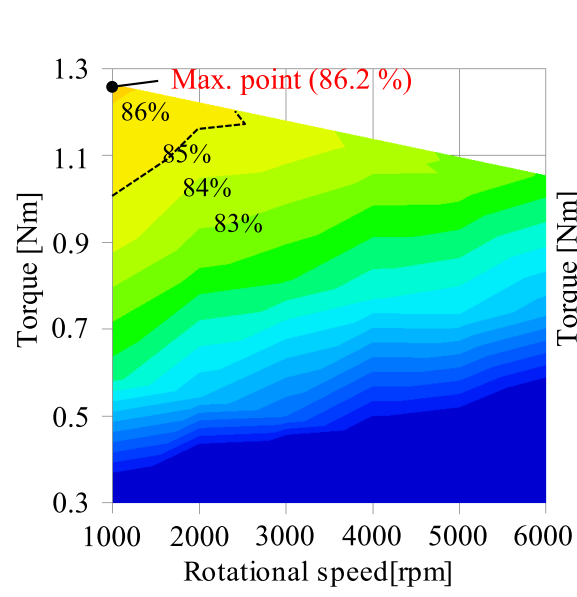

(a) Nd sintered PM model

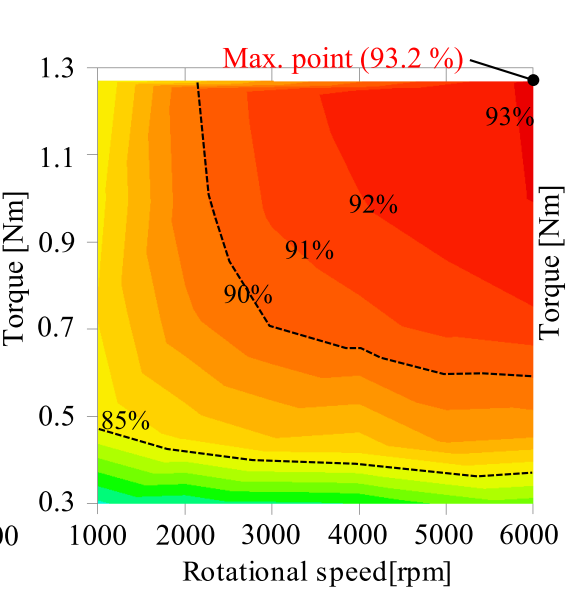

(b) Nd bonded PM model (the proposed motor)

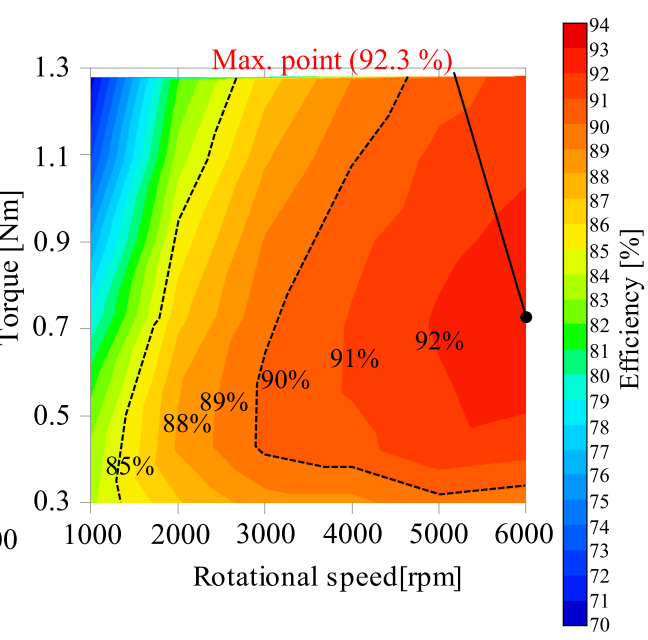

(c) Ferrite PM model

Fig. 17. Efficiency maps of three prototyped axial gap motors using different magnet materials

had the smallest iron loss of any model, but its copper loss in the heavy-load region is greater than in the other models, resulting in a total loss of $75.9 \mathrm{~W}$, which was the second largest after the model with the Nd sintered PM.

Figure 17 shows the efficiency map of each axial gap motor. As with the total loss map in Fig. 16, the efficiency map excludes the mechanical loss and evaluates the efficiency of only the electromagnetic parts of the three motors. Figure 17(a) shows the efficiency map for the model with the $\mathrm{Nd}$ sintered PM. The maximum efficiency of $86.2 \%$ occurs in the low-speed heavy-load region (1000 rpm, 3.2 Arms $/ \mathrm{mm}^{2}$ ). At higher speeds, the efficiency declines due to the effect of the eddy current loss in the Nd sintered PM. Figure 17(b) shows the efficiency map for the proposed motor using the $\mathrm{Nd}$ bonded PM. The maximum efficiency of $93.2 \%$, which is the highest result among the three models, occurs in the highspeed heavy-load region $\left(6000 \mathrm{rpm}, 4.0 \mathrm{Arms} / \mathrm{mm}^{2}\right)$. This high efficiency is due to the fact that the total loss is minimal. The above results confirm experimentally that the proposed model can achieve a high efficiency exceeding $90 \%$ over a wide range in the high-speed heavy-load region, which is the target of this study.

Figure 17(c) shows the efficiency map for the ferrite PM model. The maximum efficiency of $92.3 \%$ occurs in the highspeed medium-load region (6000 rpm, $4.0 \mathrm{Arms} / \mathrm{mm}^{2}$ ). Since the eddy current loss in the magnet is almost non-existent, the efficiency is high, especially in the high-speed region. However, as can be seen from the total loss map in Fig. 16(c), losses increase in the heavy load region due to copper loss. This is why efficiency is highest in the light- to medium-load region. The ferrite PM model achieved the highest efficiency among the three models across a wide range of the light-load region.

\section{Summary}

In this paper, we focused on axial gap motors, which are constant-torque motors with a flat shape for industrial use. Using 3D-FEA analysis and experiments on prototype motors, we confirmed that our proposed axial gap motor employing the $\mathrm{Nd}$ bonded PM could achieve high efficiency over a wide range in the high-speed heavy-load region. As a comparison, we also analyzed and tested a prototype axial gap motor employing a Nd sintered PM and one employing a ferrite PM. In both the 3D-FEA analysis and the prototype motor experiments, the proposed axial gap motor employing the $\mathrm{Nd}$ bonded PM shows the highest efficiency of all the models, reaching an efficiency of $93.2 \%$ (6000 rpm, 4.0 Arms $/ \mathrm{mm}^{2}$ ). In addition, our proposed model achieves high efficiency of $90 \%$ or more across a wide range of the target high-speed heavy-load region.

\section{References}

( 1 ) S. Kahourzade, A. Mahmoudi, N. Abdul Rahim, and H.W. Ping: "Sizing Equation and Finite Element Analysis Optimum Design of Axial-Flux Permanent-Magnet Motor for Electric Vehicle Direct Drive", in Proc. of IEEE International Power Engineering and Optimization Conference , pp.1-6 (2012)

( 2 ) Y. Okumura, M. Sanada, S. Morimoto, and Y. Inoue: "Study of Characteristics of Various Structures in Axial-gap PM Motors", in Proc. of IEEE International Conference on Electric Machines - ICEM2012, Sapporo, pp.1-6 (2012)

( 3 ) M. Aydin and M. Gulec: "A New Coreless Axial Flux Interior Permanent Magnet Synchronous Motor With Sinusoidal Rotor Segments", IEEE Trans. on Magnetics, Vol.52, No.7 (2016)

( 4 ) W. Deng and S. Zuo: "A New Coreless Axial Flux Interior Permanent Magnet Synchronous Motor With Sinusoidal Rotor Segments", IEEE Trans. on Magnetics, Vol.52, No.7 (2016)

( 5 ) Y. Aoyama, K. Miyata, and K. Ohashi: "Axial Force and Vibroacoustic Analysis of External-Rotor Axial-Flux Motors", IEEE Trans. on Industrial Electronics, Vol.65, No.3, pp.2018-2030 (2018)

( 6 ) K. Yamazaki, M. Shina, M. Miwa, and J. Hagiwara: "Investigation of Eddy Current Loss in Divided Nd-Fe-B Sintered Magnets for Synchronous Motors Due to Insulation Resistance and Frequency", IEEE Trans. on Magnetics, Vol.44, No.11, pp.4269-4272 (2008)

( 7 ) J. Li, D. Choi, C. Cho, D. Koo, and Y. Cho: "Eddy-Current Calculation of Solid Components in Fractional Slot Axial Flux Permanent Magnet Synchronous Machines", IEEE Trans. on Magnetics, Vol.47, No.10, pp.42544257 (2011)

( 8 ) X. Dai, Q. Liang, J. Cao, Y. Long, J. Mo, and S. Wang: "Analytical Modeling of Axial-Flux Permanent Magnet Eddy Current Couplings With a Slotted Conductor Topology", IEEE Trans. on Magnetics, Vol.52, No.2, p.15 (2016)

( 9 ) T. Arakawa, M. Takemoto, S. Ogasawara, K. Inoue, O. Ozaki, H. Hojo, and H. Mitani: "Examination of an Interior Permanent Magnet Type Axial Gap Motor for the Hybrid Electric Vehicle", IEEE Trans. on Magnetics, Vol.47, No.10, pp.3602-3605 (2011)

(10) A. Nihonyanagi, M. Takemoto, and S. Ogasawara: "Examination to Make Eddy Current Loss Density Uniform in Divided Nd-Fe-B Magnets of a High Torque Density Motor with Axial-gap Structure", in Proc. of the 2015 IEEE 
international Future Energy Electronics Conference (IFEEC2015), p.6 (2015)

(11) K. Chiba, S. Chino, M. Takemoto, and S. Ogasawara: "Fundamental Analysis for a Ferrite Permanent Magnet Axial Gap Motor with Coreless Rotor Structure", in Proc. of IEEE International Conference on Electric Machines ICEM2012, Sapporo, pp.1-6 (2012)

(12) W. Zhao, T.A. Lipo, and B. Kwon: "Comparative Study on Novel Dual Stator Radial Flux and Axial Flux Permanent Magnet Motors With Ferrite Magnets for Traction Application", IEEE Trans. on Magnetics, Vol.50, No.11, p.4 (2014)

(13) G.D. Donato, F.G. Capponi, G.A. Rivellini, and F. Caricchi: "Integral-Slot Versus Fractional-Slot Concentrated-Winding Axial-Flux Permanent-Magnet Machines: Comparative Design, FEA, and Experimental Tests", IEEE Trans. on Industry Application, Vol.48, No.5, pp.1487-1495 (2012)

(14) D. Wu and Z.Q. Zhu: "Design Tradeoff Between Cogging Torque and Torque Ripple in Fractional Slot Surface-Mounted Permanent Magnet Machines", IEEE Trans. on Magnetics, Vol.51, No.11, p.4 (2015)

(15) G. Heins, T. Brown, and M. Thiele: "Statistical Analysis of the Effect of Magnet Placement on Cogging Torque in Fractional Pitch Permanent Magnet Motors", IEEE Trans. on Magnetics, Vol.47, No.8, pp.2142-2148 (2011)

(16) K. Sone, M. Takemoto, S. Ogasawara, K. Takezaki, and W. Hino: "Examination for the Higher Efficiency in a Ferrite Permanent Magnet $10 \mathrm{~kW}$ In-Wheel Axial-Gap Motor with Coreless Rotor Structure", in Proc. of the 2014 IEEE Energy Conversion Congress and Exposition (ECCE 2014), pp.5885-5892 (2014)

(17) Y. Yoshida, K. Nakamura, and O. ichinokura: "Consideration of Eddy Current Loss Estimation in SPM Motor Based on Electric and Magnetic Networks", IEEE Trans. on Magnetics., Vol.48, No.11, pp.3108-3111 (2012)

(18) L. Wu, R. Qu, and D. Li: "Reduction of Rotor Eddy-Current Losses for Surface PM Machines With Fractional Slot Concentrated Windings and Retaining Sleeve", IEEE Trans. on Magnetics., Vol.50, No.11, p.6 (2014)

(19) S.S. Nair, J. Wang, L. Chen, R. Chin, I. Manolas, and D. Svechkarenko: "Prediction of 3-D High-Frequency Eddy Current Loss in Rotor Magnets of SPM Machines", IEEE Trans. on Magnetics., Vol.52, No.9, p.10 (2014)

(20) B. Wang, Yanliang, and L. Xu: "A Novel PMSM with 3-Dimensional Magnetic Circuit using SMC Core", IEEE Trans. Electrification Conference and Expo, p.5 (2017)

(21) S. Sun, K. Yang, H. Zhang, F. Jiang, and L. Tang: "A study on a novel multistage-AFPM with modular stator core made of different materials of silicon steel and SMC", in Proc. of IEEE International Conference on Electrical Machines and Systems - ICEMS2017, Sydney, pp.1-4 (2017)

(22) F. Song, K. Iwano, and K. Oba: "A Method of Cavitation Occurrence Prediction for Water Pump of Automobile Engine", The Journal of Automotive Engineering, Vol.47, No.2, pp.567-572 (2016) (in japanese)

(23) H. Qin, H. Ni, and J. Jin: "Differential Evolutionary Modeling and Startingup Cavitation Characteristics Analysis For Multistage Centrifugal Pump", in Proc. IEEE 4th Information Technology and Mechatronics Engineering Conference (ITOEC2018), pp.412-416 (2018)

Ren Tsunata (Member) was born in Miyagi Prefecture, Japan, in

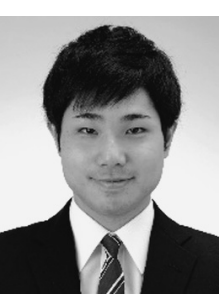
1992. He received the B.S., M.S., and Ph.D. degrees in electrical engineering from the Department of Electronics and Information Engineering, Hokkaido University, Hokkaido, Japan, in 2015, 2017, and 2021, respectively. He was with TOYOTA Motor Corporation, Aichi, Japan, in 2017-2018. Since 2021, he has been a research fellow at Okayama University, Okayama, Japan. His research interests include permanent magnet synchronous motors, variable flux motors, axial gap motors, and high power motors. Dr. Tsunata is a member of the Institute of Electrical and Electronics Engineers (IEEE) and The Japan Society of Applied Electromagnetics and Machines (JSAEM). He was a recipient of four IEEJ Excellent Presentation Awards in 2017 and 2020, respectively, and Incentive Award from JSAEM in 2020.
Masatsugu Takemoto (Member) was born in Tokyo, Japan, in 1972.

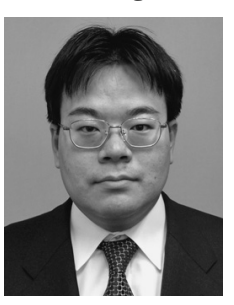
He received the B.S. and M.S. degrees in electrical engineering from Tokyo University of Science, Noda, Japan, in 1997 and 1999, respectively, and the Ph.D. degree in electrical engineering from Tokyo Institute of Technology, Tokyo, in 2005. In 1999, he joined Tokyo Institute of Technology as a Research Associate in the Department of Electrical Engineering. In 2004, he joined Musashi Institute of Technology, Tokyo, as a Research Associate in the Department of Mechanical Systems Engineering, where he became a Lecturer in 2005. In 2008, he joined Hokkaido University, Sapporo, as an Associate Professor in the Graduate School of Information Science and Technology. Since 2020, he has been with Okayama University, Okayama, Japan, where he is a Professor in the Graduate School of Natural Science and Technology. He is engaged in research on permanent magnet synchronous motors, axial gap motors, rareearth-free motors, bearingless motors, and magnetic bearings. Dr. Takemoto is a member of the Institute of Electrical Engineers of Japan (IEEJ). He was the recipient of the Nagamori Award from the Nagamori Foundation in 2017, the IEEJ Transaction Paper Award in 2005, the Prize Paper Awards from the Electric Machines Committee of the IEEE Industry Applications Society in 2011 and 2019, and the Prize Paper Award from the Electrical Machines Technical Committee of the IEEE Industrial Electronics Society in 2018. $\mathrm{He}$ has served as Secretary, Vice-Chair, and Chair of the IEEE IAS Japan chapter in 2008-2009, 2010-2011, and 2012-2013, respectively.

Satoshi Ogasawara (Fellow) was born in Kagawa, Japan in 1958.

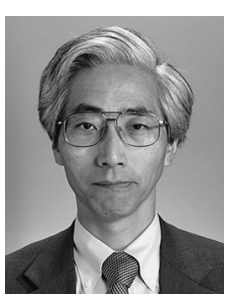
He received B.S., M.S., and Ph.D. degrees in electrical engineering from Nagaoka University of Technology, Niigata, Japan in 1981, 1983, and 1990, respectively. From 1983 to 1992, he was a Research Associate at Nagaoka University of Technology. From 1992 to 2003, he was in the Department of Electrical Engineering, Okayama University, Okayama, Japan. From 2003 to 2007, he was in the Department of Electrical Engineering, Utsunomiya University, Utsunomiya, Japan. Since 2007, he has been a Professor with the Graduate School of Information Science and Technology, Hokkaido University, Sapporo, Japan. His research interests include AC motor drive systems and static power converters. Dr. Ogasawara received the Institute of Electrical and Electronics Engineers (IEEE) Power Electronics Society Prize Paper Award in 1999 and the IEEE Industry Applications Society Committee Prize Paper Awards in 1996, 1997, 2003, and 2010. He is a fellow of the IEEJ.

Koji Orikawa (Member) received M.S. and Ph.D. degrees in elec-

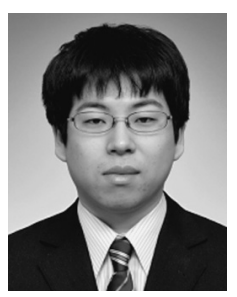
trical, electronics, and information engineering from Nagaoka University of Technology, Niigata, Japan in 2010 and 2013, respectively. From 2013 to 2016, he was a Postdoctoral Fellow at Nagaoka University of Technology. Since 2016, he has been an Assistant Professor with Hokkaido University, Hokkaido, Japan. His research interests include power conversion systems, especially DC-DC converters and high-frequency techniques for power converters. Dr. Orikawa received the IEEJ Industry Applications Society Distinguished Transaction Paper Award in 2016. He is a member of the IEEE and the IEEJ. 
Tatsuya Saito (Member) was born in Kyoto, in 1987. He received the

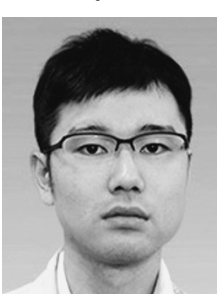
B.S., M.S., Ph.D. in material science from Tohoku University, Sendai, Japan, in 2009, 2011 and 2014, respectively. Since 2014, he has been with Sumitomo Electric Industries, Ltd., Hyogo, Japan, where he is engaged in research on soft magnetic material and powder metallurgy.
Tomoyuki Ueno (Non-member) was born in Wakayama Prefecture,

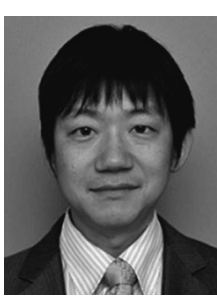
Japan, in 1976. He received the B.S. and M.S. degrees in mechanical engineering from Kobe University, Hyogo, Japan, in 1999 and 2001, respectively, and the Dr. Eng. degree in industrial innovation science from Okayama University, Okayama, Japan, in 2015. Since 2001, he has been with Sumitomo Electric Industries, Ltd., Hyogo, Japan, where he is engaged in research on soft magnetic material, ceramics material, cutting and grinding technology. Dr. Ueno is a member of the Japan Society and Power Metallurgy (JSPM) and the Japan Society for Abrasive Technology (JSAT). He was received the JSPM Award Innovatory Research in 2009, JSAT Prize Paper Award in 2012, Prize Paper Award from Machine Tool Engineer Foundation in 2012 and JSPM Award for Innovatory Development in 2013. 